\title{
Potential Effects of Teaching Strategies on Students' Academic Performance under a Trump Administration
}

\author{
Ruth Guirguis ${ }^{1}$, Jennifer Pankowski ${ }^{1}$ \\ ${ }^{1}$ Education Department, Pace University, NYC, New York \\ Correspondence: Ruth Guirguis Education Department, Pace University, NY, NY 10038, USA.
}

Received: February 17, 2017

Accepted: March 3, 2017

Online Published: March 7, 2017

doi:10.11114/jets.v5i4.2263

URL: https://doi.org/10.11114/jets.v5i4.2263

\begin{abstract}
The purpose of this meta-analysis was to explore in detail the research by Hattie (2009) in order to then re-examine it through a new lens and better understand the research and possible bias. For this study, the unit of analysis were the studies used in Hattie (2009). The criteria for inclusion were based on strategies currently implemented in NYS public school systems. Specifically, Goals, Behavioral Organizer/Advances, Mastery Learning, Meta-Cognitive Strategies, Matching Style of Learning, Teaching Strategies, Reciprocal Strategies, Comprehensive Teaching, Co-Teaching/ Team Teaching, Problem-Solving Teaching for the purpose of Teacher Preparation. These variables were further examined to determine if they may be affected should a movement towards privatizing education in NYS take effect under a Trump administration. Effect sizes, standard error, common language effect and the number of studies were used in combination to construct a forest plot with weighted effect size. A scree plot was developed to illustrate the level of precision found in this data set and a funnel plot was used to determine both precision and the existence of potential bias. Discussion on these effective teaching strategies under a new administration is broadly discussed.
\end{abstract}

Keywords: teaching strategies, meta-analysis, academic performance, potential impact

\section{Introduction}

\subsection{Statement of the Problem}

Teaching at any level of education has the sole purpose of ensuring that all learners can acquire information and apply those skills. Therefore, it is incumbent upon all educators to not only know their teaching style so that teaching has a two-fold purpose where teachers teach and students learn. Consequently, knowing how your students learn and what strategies best fit your classroom and school are fundamental in the process of learning. As a result, the question that arises in education is what are those effective teaching strategies (Hightower et al., 2011). Specifically, what strategies promote student progress in any subject area (Tebabal \& Kahssay, 2011). Moreover, with the new Trump administration, will these teaching strategies continue to be prioritized when measuring student achievement in movement towards the private sector is made?

The Trump administration has stated that a $\$ 20$ billion plan will be implemented to expand both charter and private school options for low SES (Socio-Economic Status) families and students with disabilities. Nonetheless, the specifics for carrying out this plan are still not clear as well as the impact on New York State (NYS) schools, teachers and students. Nor, how teachers would need to be prepared in higher educational programs with this change. Currently, educators are on unstable ground with discussions occurring over educational decision at the state and/or federal level as well as which policies will take precedence at either level. With a confirmed Secretary of Education, educators worry that the universal use of effective evidence based teaching strategies will be negatively affected as these strategies are proven to be successful in the public system rather than private system.

\subsection{Importance of the Problem}

The plan to leave many mandated educational decisions to the state, can lead to a lack of oversight in the use of teaching strategies with statistical evidence of effectiveness in teaching pre-K-12, as outlined in this meta-analysis. Secondly, it has been stated under the Trump administration, that the possibility of a voucher system would be developed. This system would allow for tax credits to companies who offer scholarships to those students who look for non-public schools to attend. According to Trump's educational plan, students will receive $\$ 12,000$ each in "school 
choice funds," as long as states also provide a combined $\$ 110$ billion from their education budgets. This again promotes the concept of private education, which is not where the majority of NYS educators go to for employment. Nonetheless, whether this system will be a mandated system is yet to be seen. Thus, what can be implied from the potential implementation of this system and other policies is that any additional funds that can be given to schools in high need areas may not. The concern for educators statewide is the financial influence this may have on struggling public schools and the potential systemic discrimination that may occur. Private schools and charter admit and reject students based on previously set criteria. These criteria foe exclusion is composed of, but not limited to; challenging behaviors, severe developmental delays, and some physical disabilities.

The American Federation of Teachers (2016) states that the Trump administration could use the $\$ 20$ billion school choice plan at the expense of Title I funds. This would affect the funding that would go into professional development, training, and teacher implementation of current evidence-based effective teaching strategies. As these strategies may not be of primary practice in private schools. Concurrently, this may affect public school services due to cost they will acquire in having to pay private and charter schools for local students who decided to go to those schools instead. Therefore, this leads to reflect on whether this plan is true to the law of allocating a Free and Appropriate Public Education (FAPE) for NYS students.

\subsection{Uncertainties in the Public Education System in NYS}

A further trepidation among educators is how to ease and successfully teach those students who are undocumented. Will they be able to teach and execute the same teaching evidence-based strategies and services in a private school under a new educational secretary who has a very different plan than previous administrations? If these students, do not have access to a voucher due to their undocumented status, will they also lose the opportunity to interventions such as Universal Pre-Kindergarten, Head Start, and Early Intervention programs, which offer recognized success in closing the achievement gap for English Language Learners (ELLs), low SES students and students with disabilities (Guralnick, 2005). Currently, there is so much uncertainty and questions that need to be addressed in order to prepare teachers to effectively teach under this new administration, but not a lot of transparency has been obtainable. The following meta-analysis examined the teaching strategies currently implemented for student success and their effectiveness. The paper will then revisit those strategies under the new administration to determine how potentially applicable they may be if the public school system in NYS is heavily impacted by a new private and charter school system.

\subsection{Strategies Used in Public Schools Today}

In education, there are many strategies that are implemented and strongly supported as preferred evidence based teaching methods. Hattie (2009) states that an effective teaching strategy is one that can include "explanation, elaboration, plans to direct task performance, sequencing, drill repetition, providing strategy cues, domain-specific processing and clear instructional goal" (p. 236). From the several different methods that are in place in many school the following were taken to reconfigure effect sizes; behavioral organization, mastery learning, metacognitive, matching styles, teaching strategies, reciprocal teaching, comprehensive teaching reform, co-teaching/team teaching and problem solving teaching. These approaches were scattered throughout two chapters in Hattie (2009) Visible Learning: A synthesis of over 800 meta-analyses relating to achievement (p. 162,201). The purpose of focusing on these strategies was to reflect if the effect size would change by grouping these methods differently than the way in which the author had.

\section{Method}

\subsection{Selection Criteria}

In his work, Hattie (2009) indicated that the original researchers did not implement a criterion for including or excluding studies. These studies were chosen by Hattie (2009) to investigate the effects on teaching contributions. These same studies were thus chosen to conduct this new meta-analytical review. As a result, the current researchers of this study did not need to include any moderators or code of these selected studies but two distinct variables were used to include and exclude studies used in this analysis. The researchers conducted this meta-analysis using STATA.

As previously stated, Hattie (2009) does not explain how the decision was made to separate the different type of teaching strategies into these two different chapters, therefore a selection of common and more familiar methods described in this study were selected. The effect sizes (ES) and common language effect (CLE) for these strategies were based on data already described by Hattie (2009) and used during the selection criteria. A forest plot, funnel plot, and scree plot were created in the reconfiguration of effect sizes and to test against publication bias, effect sizes, and effects of teaching strategies discussed in this study (See Appendix A). 


\section{Results}

\subsection{Part 1 of Meta-analysis Results}

Results are summarized in Table 1 and are presented in two parts. Results of the strategies reconfigured illustrate a combination of teaching methods from part I and part II of Hattie (2009). From this newly created list, a forest plot was developed (Figure 1). From part I the methods selected included; goals, behavioral organization, mastery learning, metacognitive and matching styles where chosen and from part II: teaching strategies, reciprocal teaching, comprehensive teaching reform, co-teaching/team teaching and problem solving teaching were selected.

\subsection{Effective Modeling}

An effective teaching model focuses on "teaching and learning intentions and success criteria from the challenge and purpose of the lesson" (Clarke, 2001; Clarke, Timperley, \& Hattie 2003, p. 161). Hattie (2009) stated that there are important points that are vital to learning intentions and in planning. First, teachers need to be receptive that students work at different levels and must allocate appropriate work time based on the intended instructional material. Secondly, grouping activities and learning intentions accordingly by the teacher contribute to a better understanding for the student. Lastly, students may learn other points that were not intended for (teachers need to be aware of this unintended possibility) (Hattie, 2003 p. 163). These findings were based on Hattie's (2009) synthesis of 365 meta-analyses of the contributions from teaching approaches. Overall the total for all attributes from teaching yielded an effect size $E S=.42$ and a common language effect $C L E=30 \%$.

\subsection{Goals and Behavioral/Advance Organizer}

Goals are important and critical in achieving a better student performance. This is based on the eleven meta-analyses that were conducted with an $E S=.56$ and a $C L E=40 \%$ and number of effects equaling 820 . The meta-analysis showed a relationship between goals and student performance. Hattie (2009) states that goals "inform individuals as to what type or level of performance is to be attained so that they can direct and evaluate their actions and efforts accordingly" (p.164).

According to Stone (1983) behavioral/advance organizers are "bridges from the reader's previous knowledge to whatever is to be learned" (p.167). Stone (1983) also states that these organizers were associated with better retention and increased learning of new teaching material in particular. Hattie gathered eleven meta-analyses that yield an $E S$ $=.41$ and a $C L E$ of $29 \%$, which is approaching a medium effect. Although the effect size for organizer is low to medium, the more time they are used the more the effect size increases (Luiten, Ames, \& Ackerman, 1980).

\subsection{Mastery Learning and Meta-cognitive Strategies}

Mastery learning is described as the claim that all students can learn when clear explanations are given during a concept that is being introduced or reviewed. According to Willett, Yamashita, \& Anderson (1983) mastery learning had not only had the highest effects as far as teaching strategies but it was also the most successful strategy at all educational levels. Specifically, stating that in elementary school it had an effect of $E S=.94$, in high school $E S=.72$ and in college $E S$ $=.65$, all having a high to very high effect (Guskey \& Gates, 1986). Overall there have been nine meta-analyses with $E S$ $=.58$ and a $C L E=41 \%$ (Hattie, 2009).

The fourth strategy selected was meta-cognitive strategies. According to Hattie (2009) meta-cognitive strategies involves a higher order thinking in learning. The effect size in the meta-analysis by Hattie (2009) was high: two meta-analyses, $E S=.69$ and a $C L E=49 \%$. The last teaching strategy was matching styles of learning to each student. Dunn, Griggs, Olson, Beasley, \& Gorman (1995) stated that students with stronger learning styles "such as auditory, visual, tactile, or kinesthetic styles showed greater academic gains as a result of congruent instructional interventions" (p. 195). This strategy had an $E S=.41$ and $C L E=5 \%$. Although this effect size is small, Hattie (2009) attributed it to the difficulty involved in discerning the meaning of some meta-analyses. There are different attributes that educators seem to confuse and as a result, there appears to be an overlap between learning styles and learning strategies. Another major difficulty in distinguishing studies with learning styles was that many meta-analyses reported on the learning styles with achievement and "thus are neither aptitude-treatment interactions nor learning styles intervention" (Hattie, 2009 p.195). Because of the struggle in finding and or filtering studies that reported on matching learning styles as a main focus, the $E S=.41$ may seem as if this strategy is not very important since the effect is barely approaching a moderate effect size. There has been total of eight meta-analyses on the subtopic of learning styles with a $C L E=5 \%$. 
Table 1. Summary Information from the Meta-Analyses on the Contributions from Teaching Approaches

\begin{tabular}{lllll}
\hline Study & ES & \multicolumn{1}{c}{ [95\% Conf. } & Interval] & $\%$ Weight \\
\hline Goals & 0.56 & 0.448 & 0.672 & 10.11 \\
Behavioral Org/Advances & 0.41 & 0.332 & 0.488 & 10.24 \\
Mastery Learning & 0.58 & 0.472 & 0.688 & 10.13 \\
Meta-Cognitive Strategies & 0.69 & 0.335 & 1.045 & 8.32 \\
Matching Style of Learning & 0.41 & 0.379 & 0.441 & 10.34 \\
Teaching Strategies & 0.6 & 0.486 & 0.714 & 10.1 \\
Reciprocal Strategies & 0.74 & 0.738 & 0.742 & 10.36 \\
Comprehensive Teaching & 0.22 & 0.218 & 0.222 & 10.36 \\
Co-Teaching/ Team Teaching & 0.19 & 0.078 & 0.302 & 10.11 \\
Problem-Solving Teaching & 0.61 & 0.461 & 0.759 & 9.93 \\
\hline D+L pooled ES & 0.497 & 0.266 & 0.728 & 100 \\
\hline
\end{tabular}

Note. Heterogeneity chi-squared $=1.4 \mathrm{e}+05$ (d.f. $=9) \mathrm{p}=0.000$

I-squared (variation in ES attributable to heterogeneity) $=100.0 \%$

Estimate of between-study variance Tau-squared $=0.1341$

Test of $\mathrm{ES}=0: \mathrm{z}=4.22 \mathrm{p}=0.000$

Scree Plot of Study Precision

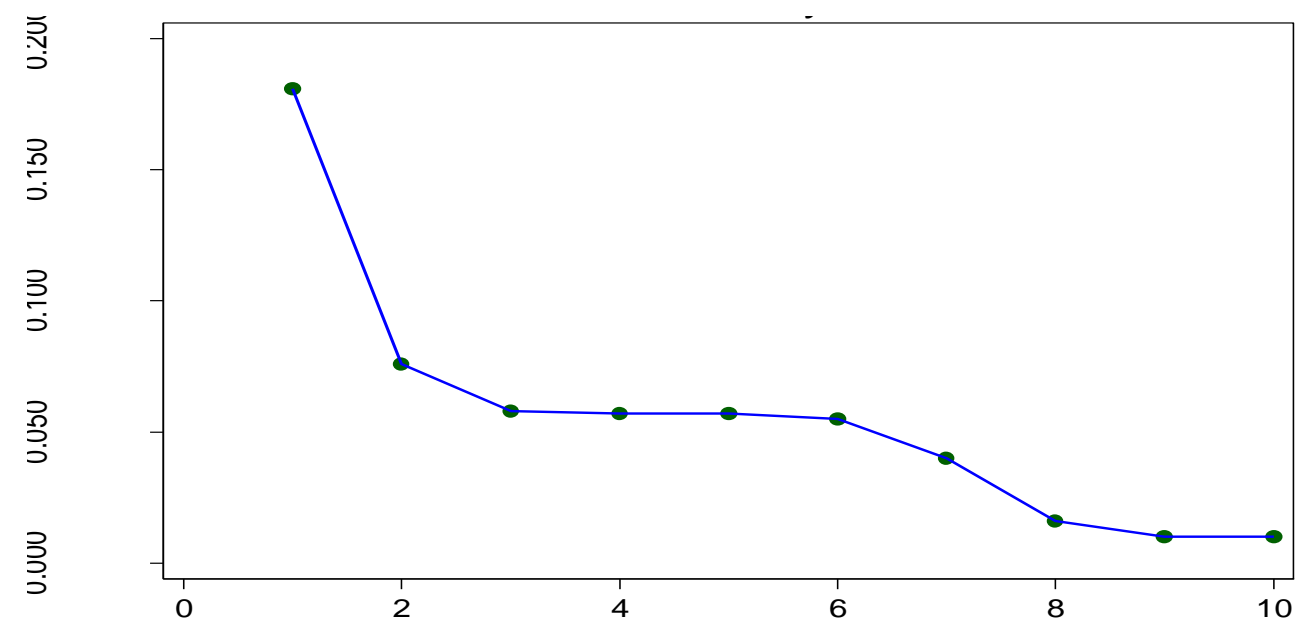

Figure 1. Studies Sorted by SE of Effect Size

\subsection{Part 2 of Meta-analysis Results}

Part two also emphasized the contributions that come from teaching approaches. The first was teaching strategies, which has a large range of different methods. We were particular surprised that Hattie (2009) would label this subtopic teaching strategies when the overall concept of the meta-analysis is Teaching Strategies. Explanations, elaboration, and plans to direct task performance are key components in an effective teaching model. Based on Swanson and Hoskyn (1998) modeling from the teacher, proper questioning and demonstrations of procedures and multi-process instructions are instructional components that are successful when teaching. Based on Hattie (2009) meta-analysis that $E S=.60$ and the $C L E=42 \%$.

\subsection{Reciprocal Teaching}

Reciprocal teaching refers to the process of teaching cognitive strategies to increase the learning abilities in students. The object of this strategy is for teachers to have students summarize, predict and clarify a concept that has been taught/learned (Hattie, 2009). There were two meta-analyses that were conducted and both analyses had a very high effect of $E S=.74$. Both meta-analyses also suggested that there was no effect difference between grade level, size of grouped students, different strategies taught by either the teacher or the investigator (Rosenshine \& Meister, 1994). The $C L E=52 \%$ and ranked ninth in the strategies listed by Hattie (2009). Borman, Hewes, Overman and Brown (2003) stated that based on their meta-analysis they found that many comprehensive teaching reform programs were being ramped up at many school districts. Superintendents and school officials believe that these types of school reforms implemented by classroom teachers are a way of improving classroom teaching (Hattie, 2009). Yet, when Hattie (2009) conducted a meta-analysis on the teaching reforms he found an extremely small effect size of $E S=.22$ and a $C L E=$ $15 \%$. 


\subsection{Co-teaching, Team Teaching}

Murawski and Swason (2001) looked at co-teaching and team teaching with special and regular education classroom teachers. Although many inclusion classrooms follow the team and or co teaching model as a positive way to mainstream special education students who need assistance in a regular education classroom, the effect size is very low. Hattie (2009) reported that there are very few studies that focus on this strategy that may attribute to the low $E S=.19$ and a $C L E=13 \%$.

\subsection{Problem Solving}

Problem solving teaching was very interesting since it had a moderately high effect of $E S=.61$ and a $C L E=43 \%$. Nonetheless, Hattie (2009) separates problem-solving teaching from problem solving learning and the way in which this type of method is measured is through the learning and performance of the students. Hembree (1992) makes a direct link between problem solving teaching and students' skill performance specifically in math. Instead of including problem based learning as a strategy, problem based teaching was selected to reconfigure the list of strategies without looking at problem based learning.

\section{Discussion}

The findings of this meta-analysis illustrate that the subtopics outlined for the new topic of Strategies for Teacher Preparation offer an overall effect size of $E S=.0497$, which based on the work of Hattie is evidence of a medium effect. Specific subtopics do however, boast more compelling effect sizes, these being Meta-Cognitive Strategies ES = .69 and Reciprocal Strategies $E S=.74$, which are effect sizes approaching the zone of desirable effects.

While the average effect size does illustrate a medium effect, two subtopics the authors believe should be considered in this topic of Strategies for Teacher Preparation do illustrate low levels and developmental levels of effect, these are Co-teaching/ Team Teaching $E S=.19$ and Comprehensive Teaching $E S=.22$. Based on the studies and the subsequent data collected, these two elements are teaching strategies that have not demonstrated statistically significant effects. There was a high degree of variation based on the $I^{2}$ value of $100.0 \%$, which reveals a large amount of heterogeneity that was highly significant, $p<.001$

\subsection{Implications for Teachers}

After reviewing several effective teaching strategies, the question for educators is whether these strategies can be implemented under an administration that looks to privatize education. The following sections take each aforementioned teaching strategy and examines it under the lens of a Trump administration and its predicted practicality. The first strategy looked at teachers' receptiveness towards the different levels in which students work at. This first strategy also looks at allocating appropriate work time based on the intended instructional material. Under the new administration a new curriculum nor discussion of revision of standards and/or standardize assessments have taken place. However, if the Trump administration succeeds in privatizing schools, little room for curriculum changes or curriculum based assessment can be accommodated as each educator would need to follow what each private school decides to use. In NYS, criterion based assessments are key in promoting and monitoring progress by each teacher depending on their students' needs.

Additionally, differentiation will be impacted as according to a 2015 United Federation of Teachers (UFT) research report, this will be of great challenge as charter schools do not take in much of our high need students. Specifically, those with a diagnosed disability, low SES, and ELLs. Choosing similar students to be part of a school, will decrease the need for teachers to correctly practice differentiation, accommodation, and modification of instruction. The UFT (2015) report indicates that the charter school sector in New York City has ignored its moral and legal duty to enroll a representative segment of the neediest students in New York City. This report suggests charters schools continue to enroll a smaller percentage of English Language learners, special education students, and other categories of needy students than local public schools (2015). The New York City Department of Education is currently the largest district in the US and has been the most progressive concerning teacher perception and differentiation in everyday teaching. Hence, being receptive to needs of all students may become a skill that will not as practiced daily in a privatized sector. The skill of a teacher's perception in recognizing and accommodating various learning styles for a diverse group of students may not be routinely exercise in charter and private school settings as they will most likely be composed of an academically homogeneous type of student body.

Accountability is yet another issue as any one private/charter school can implement their own curriculum. The consistency, validity, and reliability in holding teachers and administrators accountable is disquieting. Currently, charter schools and private schools do not have the same measures of liability that public schools faced today in NYS. The concern of inconsistent and biased assessments would be problematic in a very culturally diverse system and would lead to additional turmoil. 


\subsection{Special Education in NYS}

Hattie (2009) describes setting goals for students as a key teaching strategy in academic achievement aspect. Under a Trump administration, goal setting can become perplexing, as goals are a part of every child who receives special educational or intervention services. It can become a greater problem as charter schools take in the least amount of students with Individual Educational Plans (IEP), thus teaching to key short and long term goals will be a skill that teacher may not need to implement. Furthermore, the preparation of behavioral assessments, behavioral intervention plans, and positive behavioral systems could be reduced, due to selection of students entering private schools. Currently, the majority of IEP students attend public schools, a framework that may be affected as most students with and IEP or who is at risk may have public schools as their only choice where many educators would not end up being employed. Having more private schools opens jobs in the private sector leaving public education to then be the special education providers and the practice of teaching to emotional, behavioral and social goals may not be a skill additionally required by all new educators under a private sector. Thus, marginalizing exceptional students and educators with a special education background. As a result, a disservice can arise from being a privatized system and exercising a "one size fits all" and uniform goals in a multicultural state. This will decrease the opportunity for educators to adopt a teaching style that fits them and students' leaning styles. As well as choosing different strategies and philosophies such as a behaviorist approach, constructivist approach or an instructivist approach for teaching different students. Goals, as a strategy will most likely not be as effective when teachers will have fewer opportunities to individualize learning. Teaching through pre-set goals and standards may lead teaching to become more didactic and not support a holistic and equitable development of the child nor form of teaching.

\subsection{Inclusion}

Inclusion is a philosophy that teachers hold as a belief and way of thinking, acting that allows all students' values to be accepted and feel safe. Inclusion allows all learners to experience an educational environment welcoming of all needs. Inclusion also allows students to interact with peers who have a different perspective and approach to learning. Will this philosophy have a place in the Trump administration, with a culture of exclusion? Currently, many of our students who for example who are of illegal status will not benefit nor flourish from an inclusive community that consciously evolves to meet the changing needs of students. It is an educators' responsibility to meet the needs of all students, but what if regulations and sanctions are placed on teachers and schools? This can negatively impact the special education system with the idea of separating students and given parents "choices". Nonetheless, can strategies such as reciprocal teaching and changing comprehensive teaching be implemented under this current administration? The goal of these strategies are to have students summarize, predict and clarify. Strategies which can be successful under this new political administration if both permitted and encouraged with proper oversight and funding for things such as professional development. While the aforementioned strategies are effective in reaching student achievement, the policy aspect was considered into this meta-analyses as a lens to foresee some possible changes in teaching and strategies.

\subsection{Limitations}

The present meta-analysis, like most others, has several limitations. First, meta analysts do not have experimental control over previously collected data, which reduces the sensitivity of the analysis. Secondly, the overall findings of this meta-analysis are limited by the quality of the primary studies. Another perceived limitation of this meta-analysis may be that we included only published articles from Hattie (2009). Finally, our study relates to the recentness of the original meta-analysis review. However, there is a paucity of meta-analyses on the topic of potential effective teaching strategies in education and specifically as it applies under a new administration. Future research syntheses may want to include either more rigorous criteria to ensure that only recent research are included to examine if there are any differences in trends.

\subsection{Conclusion}

According to Ganyaupfu (2013) several methods, such teacher, student, and teacher-student are most valuable in the implementation of the Common Core State Standards. Teacher-Centered Methods allow students to receive instruction from their teacher. Student-centered methods allow students the opportunity to discover learning through the implementation of students' interests. Hence, learning takes on a more goal oriented approach (Ganyaupfu, 2013). Finally, a teacher-student interactive method is one used by the previous methods combined to engage the student and have periods of teacher led instruction. While this meta-analysis has explored various evidence-based teaching strategies which would allow all students to learn and develop, the major concern for the authors is, how effective will these public school strategies work under a potential private sector. This administration has developed a platform which focuses on providing parents with "choice" however, without a full understanding of this system, teaching strategies can change to meet the new secretary of education plan of school privatization. Finally, as a state composed of cultural and linguistic diversity, the choice of privatization of education can put some the at risk students to be left of as school will 
pick those students that best fit their educational framework. Therefore, limiting teachers' ability to use effective strategies such as reciprocal teaching, co-teaching, collaboration, inclusion, problem-solving strategies which hinge upon working with a heterogeneous population of students and faculty in order to gain multiple perspectives.

\section{References}

Borman, G. D., Hewes, G. M., Overman, L. T., \& Brown, S. (2003). Comprehensive school reform and achievement: A meta analysis. Review of Education Research, 73(2), 125-230. https://doi.org/10.3102/00346543073002125

Clarke, S. (2001). Unlocking formative assessment: Practical strategies for enhancing pupils' learning in the primary classroom. London: Hodder and Stoughton Education.

Clarke, S., Timperley, H., \& Hattie, J. (2003). Unlocking formative assessment: Practical strategies for enhancing pupils' learning in the primary and intermediate classroom ( $1^{\text {st }}$ N.Z. ed) Auckland, New Zealand: Hodder Moa Beckett.

Dunn, R., Griggs, S. A., Olson, J., Beasley, M., \& Gorman, B. S. (1995). A meta-analysis validation of the Dunn and Dunn model of learning-style preferences. Journal of Educational Research, 88(6), 353-362. https://doi.org/10.1080/00220671.1995.9941181

Ganyaupfu, E. M. (2013). Teaching methods and students' academic performance. International Journal of Humanities and Social Science Invention, 2(9), 29-35.

Guralnick, M. (2005). Early intervention for children with intellectual disabilities: Current knowledge and future prospects. Journal of Applied Research in Intellectual Disabilities, 18(4), 313-324. https://doi.org/10.1111/j.1468-3148.2005.00270.x

Guskey, T. R., \& Gates, S. L. (1986). Synthesis of research on the effects of mastery learning in elementary and secondary classrooms. Educational Leadership, 43(8), 73-80.

Hattie, J. (2009). Visible learning: A synthesis of over 800 meta-analyses relating to achievement. New York, NY: Routledge.

Hembree, R. (1992). Response to Critique of Meta-Analysis. Journal for Research in Mathematics Education, 23(3), 284-289. https://doi.org/10.2307/749122

Hightower, A., Delgado, R., Lloyd, S., Wittenstein, R. Sellers, K., \& Swanson, C. (2011). Improving student learning by supporting quality teaching: Key issues, effective strategies, Editorial Projects in Education, Inc., Bethesda, MD.

Luiten, J., Ames, W., \& Ackerman, G. (1980). A meta-analysis of the effects of advance organizers on learning and retention. American Educational Research Journal, 17(2), 211-218. https://doi.org/10.3102/00028312017002211

Murawski, W. W., \& Swason, H. L. (2001). A meta-analysis of co-teaching research: Where are the data? Race: Remedial and Special Education, 22(5), 258-267. https://doi.org/10.1177/074193250102200501

Rosenshine, B., \& Meister, C. (1994). Reciprocal teaching: A review of the research. Review of Educational Research, 64(4), 479-530. https://doi.org/10.3102/00346543064004479

Stone, C. L. (1983). A meta-analysis of advance organizer studies. Journal of Experimental Education, 51(4), 194-199. https://doi.org/10.1080/00220973.1983.11011862

Swanson, H. L., \& Hoskyn, M. (1998). Experimental intervention research on students with learning disabilities: A meta-analysis of treatment outcomes. Review of Educational Research, 68(3), 277-321. https://doi.org/10.3102/00346543068003277

Tebabal, A., \& Kahssay, G. (2011), The effects of student-centered approach in improving students' graphical interpretation skills and conceptual understanding of kinematical motion, Lat. Am. J. Phy. Edu., 5(2), 374-381.

United Federation of Teachers (2015). Research, analysis and policy recommendations from the UFT, retrieved 2/8/2017 from http://www.uft.org/where-we-stand/reports

Willett, J. B., Yamashita, J. J. M., \& Anderson, R. D. (1983). A meta-analysis of instructional systems applied in science teaching. Journal of Research in Science Teaching, 20(5), 405-417. https://doi.org/10.1002/tea.3660200505 


\section{Appendix A}

Teacher Preparation Strategies

\begin{tabular}{|c|c|c|c|c|c|}
\hline Effect & Studies & CLE & & ES (95\% Cl) & $\begin{array}{l}\% \\
\text { Weight }\end{array}$ \\
\hline Goals & 604 & $40 \%$ & & $0.56(0.45,0.67)$ & 10.11 \\
\hline Behavioral Org/Advance & 577 & $29 \%$ & $\rightarrow$ & $0.41(0.33,0.49)$ & 10.24 \\
\hline Mastery Learning & 377 & $41 \%$ & & $0.58(0.47,0.69)$ & 10.13 \\
\hline Meta-Cognitive Strategies & 63 & $49 \%$ & & $0.69(0.34,1.04)$ & 8.32 \\
\hline Matching Style of Learning & 411 & $29 \%$ & - ! & $0.41(0.38,0.44)$ & 10.34 \\
\hline Teaching Strategies & 5667 & $42 \%$ & & $0.60(0.49,0.71)$ & 10.10 \\
\hline Reciprocal Strategies & 38 & $52 \%$ & - & $0.74(0.74,0.74)$ & 10.36 \\
\hline Comprehensive Teaching & 282 & $15 \%$ & - & $0.22(0.22,0.22)$ & 10.36 \\
\hline Co-Teaching/ Team Teaching & 136 & $13 \%$ & $\rightarrow-$ & $0.19(0.08,0.30)$ & 10.11 \\
\hline Problem-Solving Teaching & 221 & $43 \%$ & & $0.61(0.46,0.76)$ & 9.93 \\
\hline \multicolumn{3}{|c|}{ Overall (I-squared $=100.0 \%, p=0.000$ ) } & & $0.50(0.27,0.73)$ & 100.00 \\
\hline NOTE: Weights are from rando & alysis & & & & \\
\hline
\end{tabular}

Funnel plot with pseudo 95\% confidence limits
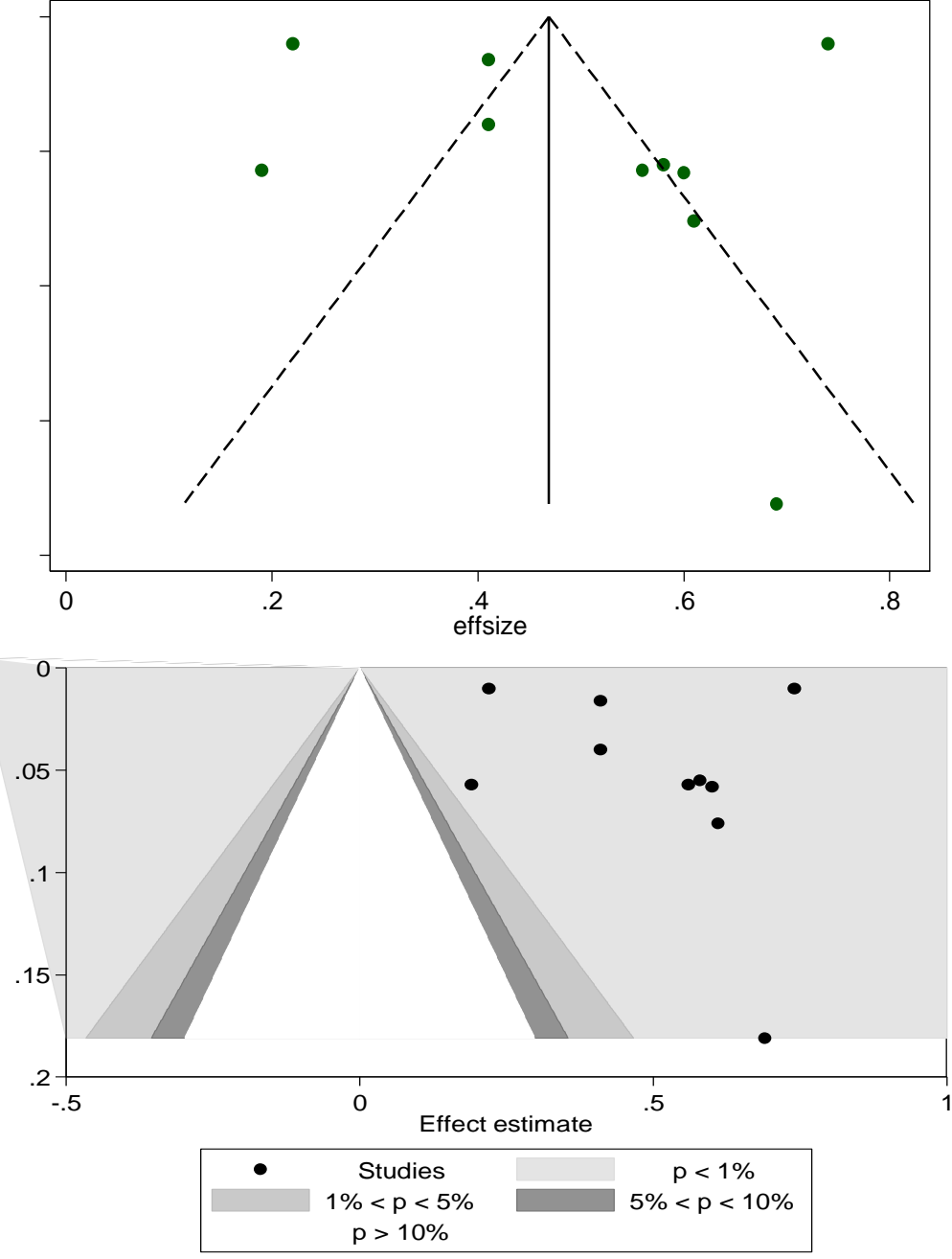

\section{Copyrights}

Copyright for this article is retained by the author(s), with first publication rights granted to the journal.

This is an open-access article distributed under the terms and conditions of the Creative Commons Attribution license which permits unrestricted use, distribution, and reproduction in any medium, provided the original work is properly cited. 